\title{
Blower Gun pellet injection system for W7-X
}

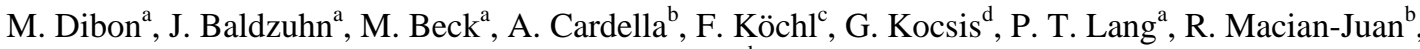 \\ B. Ploeckl ${ }^{\mathrm{a}}$, T. Szepesi ${ }^{\mathrm{d}}$ and W. Weisbart ${ }^{\mathrm{a}}$ \\ ${ }^{a}$ Max-Planck-Institute for Plasma Physics, Boltzmannstr. 2, 85748 Garching, Germany \\ ${ }^{b}$ Lehrstuhl für Nukleartechnik, TU Munich, Boltzmannstr. 15, 85748 Garching, Germany \\ ${ }^{c}$ Atominstitut, TU Wien, 1020 Vienna, Austria \\ ${ }^{d}$ Wigner RCP, RMI, P.O.Box 49, H-1525 Budapest-114, Hungary
}

Foreseen to perform pellet investigations in the new stellarator W7-X, the former ASDEX Upgrade Blower Gun was revised and revitalized. The systems operational characteristics have been surveyed in a test bed. The gun is designed to launch cylindrical pellets with $2 \mathrm{~mm}$ diameter and $2 \mathrm{~mm}$ length, produced from frozen Deuterium $\mathrm{D}_{2}$, Hydrogen $\mathrm{H}_{2}$ or a gas mixture consisting of $50 \% \mathrm{H}_{2}$ and $50 \% \mathrm{D}_{2}$. Pellets are accelerated by a short pulse of pressurized helium propellant gas to velocities in the range of $100-250 \mathrm{~m} / \mathrm{s}$. Delivery reliabilities at the launcher exit reach almost unity. The initial pellet mass is reduced to about $50 \%$ during the acceleration process. Pellet transfer to the plasma vessel was investigated by a first mock up guiding tube version. Transfer through this S-shaped stainless steel guiding tube (inner diameter $8 \mathrm{~mm}$; length $6 \mathrm{~m}$ ) containing two $1 \mathrm{~m}$ curvature radii was investigated for all pellet types. Tests were performed applying repetition rates from $2 \mathrm{~Hz}$ to $50 \mathrm{~Hz}$ and propellant gas pressures ranging from 0.1 to $0.6 \mathrm{MPa}$. For both $\mathrm{H}_{2}$ and $\mathrm{D}_{2}$, low overall delivery efficiencies were observed at slow repetition rates, but stable efficiencies of about $90 \%$ above $10 \mathrm{~Hz}$. About $10 \%$ of the mass is eroded while flying through the guiding tube. Pellets exit the guiding tube with an angular spread of less than $14^{\circ}$.

Keywords: pellet injection, plasma fuelling

\section{Introduction}

One of the main objectives during the first operational phase of W7-X will be the investigation of high density plasmas. As shown in previous experiments conducted on ASDEX Upgrade [1] and JET, pellet fuelling is the best option for reaching high plasma densities and a good fuelling efficiency. Hence, W7-X will perform pellet injection experiments, using solid hydrogen during an early operational phase. Fuelling size pellets (cylindrical, $\mathrm{d}=2 \mathrm{~mm}, \quad \mathrm{l}=2 \mathrm{~mm}$ ) are required to achieve the necessary density and a repetition rate above $10 \mathrm{~Hz}$ in order to maintain it. Additionally, the pellets have to be injected at a sufficiently high velocity to deposit the material near the core plasma. Studies showing the impact of pellet injection from both the magnetic low and high field side are planned.

Considering these requirements, the ASDEX Upgrade Blower Gun has been chosen to perform as injection system for the first operational phase of W7-X. This system has proven to work reliably during its service at ASDEX Upgrade. In order to be fit for the operation at W7-X, the injector was modified for the utilization of hydrogen as pellet material. Furthermore, it was equipped with a guiding tube, mimicking the critical segment of the high field side guiding tube. This setup underwent a complete characterization, in which different pellet materials, repetition rates and pellet velocities were tested. Critical parameters like the pellet mass, delivery reliability and the pellet velocity were measured at the exit of the Blower
Gun and after the guiding tube. By these means, the overall mass loss, transfer efficiency and speed scatter through the injector and the guiding tube were obtained.

\section{Experimental setup}

\subsection{AUG Blower Gun}

Unlike pneumatic gas guns, the blower gun accelerates the pellets by drag between the propellant gas and the pellet. This is achieved by shooting the pellet through a barrel, of which the inner diameter is slightly larger than the pellets outer diameter. This small gap allows the propellant gas to flow around the pellet, leading to a smooth speed up. Though the complete enclosure of the pellet inside the gas leads to an increased heat transfer to the pellet.

The solid hydrogen is formed inside the extrusion cryostat. It is cooled by liquid helium to a temperature of about $5 \mathrm{~K}$. This is sufficient to freeze the hydrogen or deuterium gas immediately to the wall of the cryostat. After the formation of a thin ice layer, the cryostat is slightly heated and a pneumatic piston scrapes the ice off the wall, pushing it through a narrow nozzle. By this, a thin rod of compressed hydrogen ice is created. Afterwards, the ice rod is pushed into the storage cryostat. This component is also cooled by liquid helium, but reaches a higher temperature limit of about $10 \mathrm{~K}$, due to its large mass and surface. The storage cryostat can preserve hydrogen ice up to 30 minutes and deuterium ice for over an hour. It can 
hold an ice rod with $2 \mathrm{~mm}$ diameter and $124 \mathrm{~mm}$ length. Theoretically, this allows 62 fuelling size pellets to be shot before new ice has to be produced, although some ice is lost due to the shuttle at the exit of the cryostat and the lever.

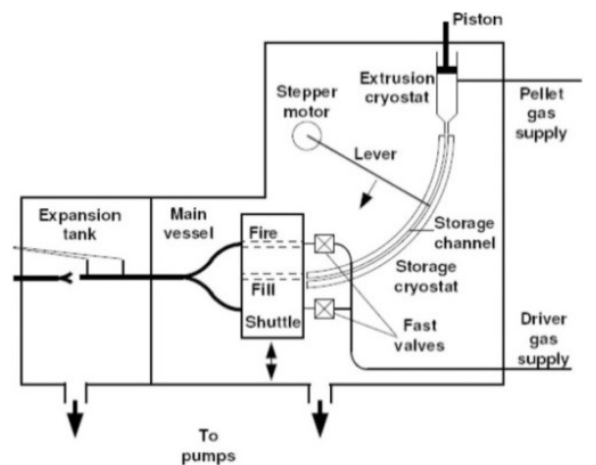

Figure 1: Scheme of the AUG Blower Gun[2]

When pellets are requested, a lever moves into the storage cryostat, cuts off the rod and pushes it out of the cryostat into the shuttle. This shuttle cuts a pellet off the ice rod and moves it in front one of the two fast gas valves. The valve opens and a short pulse of propellant gas accelerates the pellet into the barrel. While one pellet is shot, the shuttle can take up another pellet from the storage cryostat. By this two stroke mechanism, the blower gun is capable of repetition rates above $140 \mathrm{~Hz}$. The injector is closely described in reference [2].

\subsection{Guiding tube}

It is foreseen, that the W7-X setup will contain two separate pellet guiding tubes, one to the magnetic low and one to the high field side. Both will be made of stainless steel tubes with a circular crosssection. While the tube to the low field side has only one bend with a large radius, the geometry of the tube leading to the high field side is more complex. In order to dive under the vessel and access the inboard side, it is necessary to implement several bends with radii of about $1 \mathrm{~m}$. Both guiding tubes were investigated in a mock up. Because of the limited space in the laboratory, only parts of the tubes could be tested. The low field side guiding tube was represented by a stainless steel tube with an overall length of $4.44 \mathrm{~m}$, containing a straight passage of $2.51 \mathrm{~m}$ and a bend with $5.5 \mathrm{~m}$ curvature radius. For the tube to the high field side it was decided to investigate the critical part of the tube, which is foreseen to be the S-shaped segment. This was represented by a stainless steel tube, containing two bends with curvature radii of $1 \mathrm{~m}$, a $0.5 \mathrm{~m}$ long straight section between the bends and two straight segments of $1.18 \mathrm{~m}$ before and after the bends. Both tubes had a circular cross-section with an inner diameter of $8 \mathrm{~mm}$ and a wall thickness of $1 \mathrm{~mm}$. The inner surface was electro polished to avoid sharp edges. Both tubes were neither actively cooled nor directly pumped.

\subsection{Diagnostics}

Observing the pellet motion inside the Blower Gun and through the guiding tube was the main objective of the investigation. For this purpose, the experimental setup was equipped with several diagnostics to survey the pellet parameters. Two light barriers were placed at the end of the barrel, the direction of the beams looking perpendicular to the flight direction of the pellets. Another two light barriers were installed after the guiding tube. This setup allowed the time-of-flight measurement of the pellets, as well as the observation of the pellet speeds at the exit of the injector and after the passage through the guiding tube. In addition to that, the light barriers were used to count the pellets leaving either the gun barrel or the guiding tube. By this it was possible to calculate the transfer efficiency through the injector and the tube. The light barriers were used as trigger signals for the shadowgraphy cameras. These were applied to take images of the pellets in flight. Two cameras were installed, one at the barrel exit, the other after the guiding tube. They viewed perpendicular to the flight path of the pellets and were set to a short exposure time due to the speed of the pellets. This made it necessary to add lasers with diverging lenses as artificial light sources opposing the cameras.

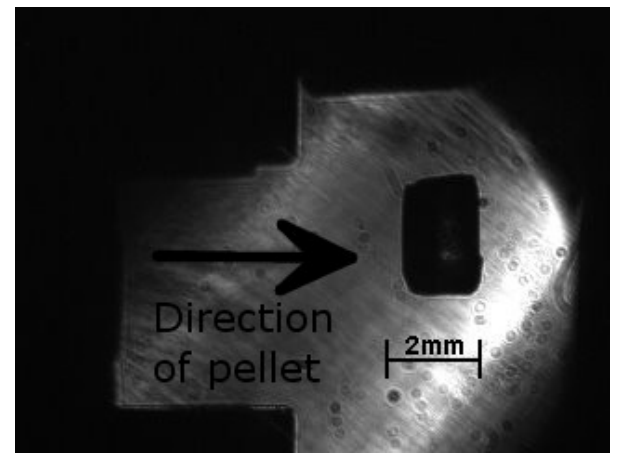

Figure 2: Shadowgraphy image of a $\mathrm{H}_{2}$ pellet at the exit of the Blower Gun

Images were used to calculate the volumes of the pellets, applying bayesian analysis [3]. From that, the mass loss through the Blower Gun and the guiding tube was determined. The exit angle of the guiding tube was measured by stretching out a strip of thin paper perpendicular to the main flight direction of the pellets. The distance between the paper and the exit of the tube was $0.221 \mathrm{~m}$. The pellets punched through the paper, leaving visible holes in the paper strip. This allowed an easy measurement of the distance between the holes and the central axis and thus the exit angle. In addition to the thermosensors and pressure gauges, which were used to monitor the systems operation, the setup was complemented by a quadrupole mass spectrometer after the guiding tube. This device allowed for the investigation of the pellet composition in case of hybrid pellets. 


\section{Experimental procedure}

First experiments were conducted using the guiding tube with the large bending radius, deuterium as pellet material and helium as propellant gas. The repetition rate was gradually increased from $2 \mathrm{~Hz}$ up to $50 \mathrm{~Hz}$ in steps of $2 \mathrm{~Hz}$. Propellant gas pressures between 0.1 MPa to 0.6 MPa were applied for every repetition rate. The pressure was increased in steps of $0.1 \mathrm{MPa}$. For every repetition rate and pressure, two ice rods were used, each delivering 30 pellets.

In a second step, the first guiding tube was replaced by the S-shaped tube. Pellet material and propellant gas were kept as before. The repetition rate was raised in steps of $2 \mathrm{~Hz}$ between $2 \mathrm{~Hz}$ and $10 \mathrm{~Hz}$. Above this rate, the increment was $5 \mathrm{~Hz}$. The gas pressures and their increment were kept the same, such as the pellet number per pressure and repetition rate. Only at $10 \mathrm{~Hz}$, it was decided to use 10 ice rods per gas pressure in order to achieve a more precise result, since this will be the preferred setting at W7-X.

The third step of testing was run using hydrogen as pellet material. Guiding tube and propellant gas were kept as before. The ranges and increments of the repetition rates and gas pressures remained unchanged, as for the pellet number. This allowed a comparison between the two different isotopes in the pellets. Furthermore, as hydrogen will be the pellet material used during the first operational phase of W7-X, the exit angle was also measured using this setup.

The fourth experiment was conducted using a gas mixture consisting of $50 \% \mathrm{H}_{2}$ and $50 \% \mathrm{D}_{2}$ for pellet formation. Guiding tube and propellant gas were kept. The increment between $10 \mathrm{~Hz}$ and $50 \mathrm{~Hz}$ was raised to $10 \mathrm{~Hz}$. The other ranges and increments regarding repetition rate and gas pressure remained unchanged. The same applied to the pellet number per setting.

\section{Results}

The pellet speeds show a clear increase with growing propellant gas pressures. Although the flow velocity of the gas doesn't very much at different pressures, the gas density near the fast gas valves depends on the pressure. This higher density is the prime factor for the higher pellet speed at greater propellant gas pressure. It can also be seen, that the increase of the pellet speeds is smaller at high pressures. The Blower Gun is designed for propellant gas pressure up to $0.6 \mathrm{MPa}$, but it can be extrapolated, that higher pressures wouldn't have much impact on the pellet speed. Another result is that the hydrogen pellets are significantly faster than the deuterium pellets. This is due to the lower density of the hydrogen ice and thus a lower pellet mass. The speed of the hybrid pellets lies between the ones for $\mathrm{H}_{2}$ and $\mathrm{D}_{2}$. Further it is observed, that the decrease of the pellet speed through the guiding tube is marginal, although the scatter of the pellet speed was distinctly higher. This was verified for both guiding tubes tested in the mock up.

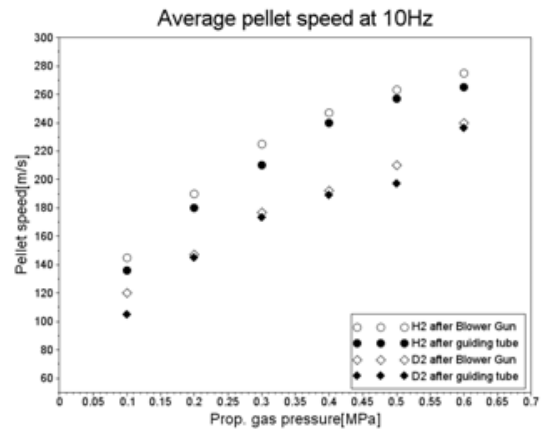

Figure 3: Pellet speeds, setup with critical guiding tube (only $\mathrm{H}_{2}$ and $\mathrm{D}_{2}$ )

As for the transfer efficiency (number of registered pellets/requested pellets) it was shown, that the guiding tube and the propellant gas pressure have nearly no noticeably influence. The efficiency through both tubes was near unity. Regarding the transfer efficiency in the Blower Gun, a strong influence of the repetition rate can be observed. At $2 \mathrm{~Hz}$, the transfer efficiency is comparatively low for all pellet materials. This effect is due to the warm shuttle. Its thermal radiation on the ice rod in the storage cryostat reduces the mechanical strength of the ice, leading to more destroyed pellets. This effect is stronger for hydrogen and thus for hybrid pellets as well. Only above $15 \mathrm{~Hz}$, all pellets reach transfer efficiencies above $85 \%$. Above $30 \mathrm{~Hz}$, the efficiencies decrease again. It is assumed, that the cause is an increased vibration of the shuttle at higher frequencies.

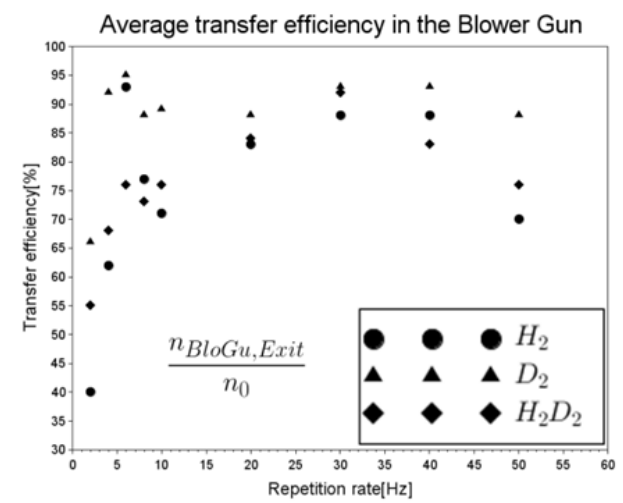

Figure 4: Transfer efficiencies in the Blower Gun

The mass loss is mainly depended on the propellant gas pressure. The higher gas density leads to larger heat loads on the pellets, resulting in a faster loss of pellet mass. This effect can be observed in the Blower Gun and the guiding tubes, indicating that a certain amount of propellant gas reaches the tubes. Both tested guiding tube geometries show very similar results concerning 
mass loss. As for the repetition rates, their influence on the pellet mass is similar as for the transfer efficiency.

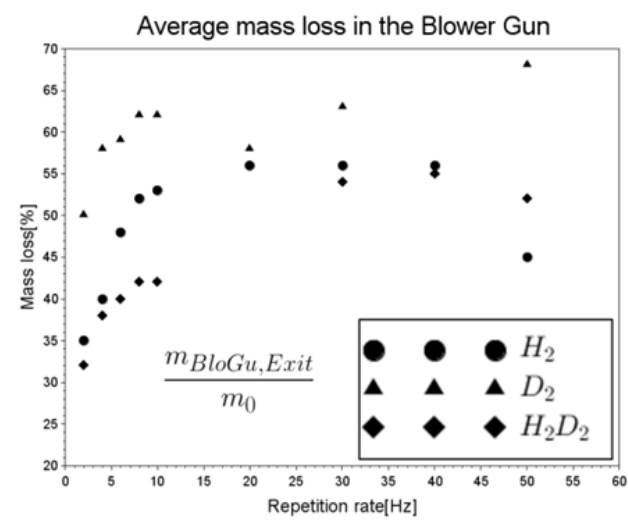

Figure 5: Mass loss in the Blower Gun

The pellet material also has a large impact on the mass loss. Deuterium has the overall highest residual mass. Hydrogen pellets in comparison loose much more material, due to the lower sublimation temperature and heat capacity of $\mathrm{H}_{2}$. The hybrid pellets show the overall lowest residual mass. It is assumed, that the hydrogen sublimates before the deuterium, forming small gas filled cavities in the pellet. These gas bubbles could, when reaching a certain pressure, blow off small pieces from the pellet, leading to a larger mass loss. The loss of pellet material is about $50 \%$ in the Blower Gun. Another 10\% of pellet mass is lost in the guiding tube, due to sublimation on the walls of the tube. These values are comparable to previous experiments conducted with the JET pellet injection system [5], but the losses are higher than with other methods of pellet injection, e.g. a centrifuge.

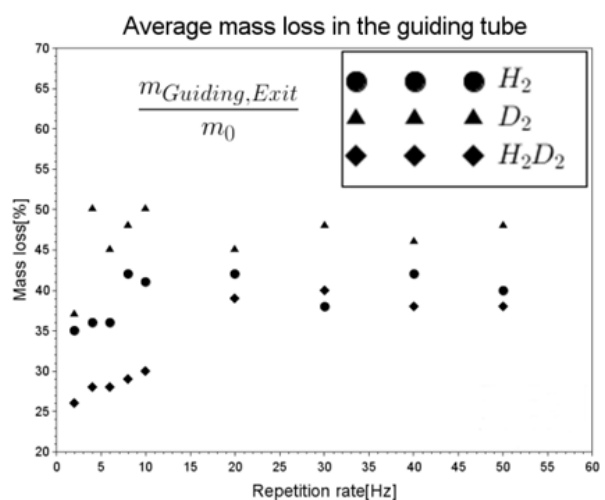

Figure 6: Mass loss in the Blower Gun and the guiding tubes

The exit angle shows no perceptible dependency on repetition rate and propellant gas pressure. The impact pattern on the paper strip indicates that the last turn in the guiding tubes has a small influence on the exit position. But, because of the circular cross-section of the tube, the pellets have no preferred exit trajectory. The overall spread angles are between $1^{\circ}$ and $14^{\circ}$.

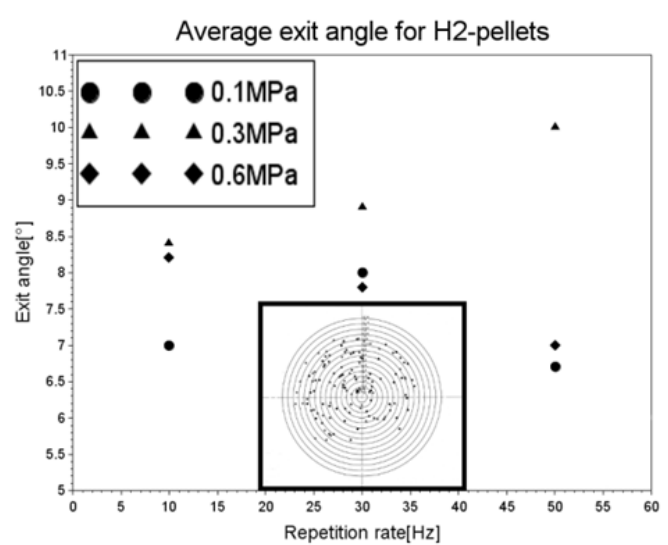

Figure 7: Average exit angle and scatter plot(inlay)

\section{Conclusion}

The Blower Gun meets the requirements of W7-X for the first operational phase. Both hydrogen and deuterium pellets can be produced reliably and accelerated to speeds between $120 \mathrm{~m} / \mathrm{s}$ and $290 \mathrm{~m} / \mathrm{s}$, depending on the propellant gas pressure and the pellet material. The transfer efficiency in the Blower Gun is mainly affected by the repetition rate whereas efficiencies around $85 \%$ can be achieved. The guiding tube has no noticeable effect on the transfer efficiency. For the mass loss it was shown, that about $50 \%$ of the initial pellet mass is lost during the acceleration in the Blower Gun, while another $10 \%$ is evaporated in the guiding tube, mainly depending on the propellant gas pressure. The off-axis spread angle lies between $1^{\circ}$ and $14^{\circ}$.

\section{References}

[1] P.T. Lang, et al., Refueling performance improvement by high speed pellet launch from the magnetic high field side, Nuclear Fusion 41, 1107-1112, 2001

[2] P.T. Lang, et al.: A pellet launcher tool optimized for the control of edge localized modes in ASDEX Upgrade H-mode plasmas, Fusion Engineering and Design 82, 1073-1080, 2007

[3] T. Szepesi, et al.: Volume measurement of cryogenic deuterium pellets by Bayesian analysis of single shadowgraphy images, Review of Scientific Instruments, Vol. 79, 2008

[4] P.T. Lang, et al., Cryogenic pellet launcher adapted for controlling of tokamak plasma edge instabilities, Review of Scientific Instruments 78, 023504, 2007

[5] P.T. Lang, et al., ELM pacing investigations at JET with the new pellet launcher, Nuclear Fusion 51, 033010, 2011 